University of Louisville

ThinkIR: The University of Louisville's Institutional Repository

College of Arts \& Sciences Senior Honors

Theses

College of Arts \& Sciences

$5-2017$

\title{
Infants' perception of faces in face-like and ambiguous images.
}

Lauren E Dale

University of Louisville

Follow this and additional works at: https://ir.library.louisville.edu/honors

Part of the Child Psychology Commons

Recommended Citation

Dale, Lauren E, "Infants' perception of faces in face-like and ambiguous images." (2017). College of Arts \& Sciences Senior Honors Theses. Paper 134.

http://doi.org/10.18297/honors/134

This Senior Honors Thesis is brought to you for free and open access by the College of Arts \& Sciences at ThinkIR: The University of Louisville's Institutional Repository. It has been accepted for inclusion in College of Arts \& Sciences Senior Honors Theses by an authorized administrator of ThinkIR: The University of Louisville's Institutional Repository. This title appears here courtesy of the author, who has retained all other copyrights. For more information, please contact thinkir@louisville.edu. 
Infants' Perception of Faces in Face-Like and Ambiguous Images

\section{By}

\section{Lauren Elizabeth Dale}

Submitted in partial fulfillment of the requirements

for Graduation summa cum laude

and

for Graduation with Honors from the Department of Psychological and Brain Sciences

University of Louisville

May, 2017 


\begin{abstract}
Faces are special to infants. Infants look longer at faces than objects, human faces than non-human faces, and upright faces compared to inverted faces. The objective of this study was to see if infants demonstrated signs of pareidolia. Pareidolia is the ability to see a face in a nonface object, such as a face in a cloud. Previous research has shown that adults see faces in everyday objects, but less is known about infants' perception of such images. In the present study, infants 7-9 months old $(N=47)$ were tested. Infants were shown eight images, which adults had ordered from most face-like to least face-like, over 16 trials. An upright and inverted version of each image was presented side by side to infants on each trial. Infants' looking times to each side was recorded. It was reasoned that infants would show a reliable preference for the upright version of a stimulus if they perceived a face in that stimulus. It was found that infants showed an upright preference for three of the eight stimuli, two that were deemed most face-like (i.e., a color photo of a female face, and a black and white schematic face) and one grouped as moderately face-like (i.e., a black and white Mooney image that could be perceived as either a face or a man playing a saxophone). From these results, we conclude that infants can perceive a face, but only in images that are the most face-like. The results suggest that infants may experience some form of pareidolia but not to the same extent as adults do. Future studies should further investigate the development of pareidolia infants and children.
\end{abstract}


Infants' Perception of Faces in Face-Like and Ambiguous Images

Faces are special to adults. It is generally believed that adults are experts in face recognition. In recognizing faces, adults use several processes (Maurer, Le Grand, and Mondloch 2002). Configural processing involves organizing and relating facial features to one another (Ichikawa et al., 2011), whereas featural processing involves attending to individual facial features, but not to the relations among those features. It is believed that processing a face configurally is a marker of expertise in face processing (Maurer, Le Grand, and Mondloch (2002). Adults process upright faces configurally but process inverted faces featurally (e.g., Diamond \& Carey, 1986; Maurer, Le Grand, \& Mondloch, 2002; Rakover, 2013).

Adults also have the capability to see faces in everyday objects around them (Ichikawa, Kanazawa, \& Yamaguchi, 2011; Kato \& Mugitani, 2015). For example, sometimes we can see a face in the clouds or in the arrangement of pepperoni on our pizza. This phenomenon is known as pareidolia. The exact reason for pareidolia is unknown, but it is believed that it is related to adults' expertise in face processing and some form of configural processing. Ichikawa et al. also believe it is related to adults' tendency to perceive a pair of eyes in non-face objects (Ichikawa et al., 2011).

Faces are also special to infants. It is obvious to anyone who has interacted with infants that they develop a fascination with faces at an early age. Research indicates that during the first year of life infants show a preference for looking at human faces over animal faces (Di Giorgio, Méary, Pascalis \& Simion, 2013; Jakobson, Umstead \& Simpson, 2016). Infants also prefer faces over other objects; when presented pictures of faces and toys simultaneously, infants direct their gazes toward faces before other stimuli and faces hold their attention longer (DeNicola, Holt, Lambert \& Cashon, 2013; Gliga, Elsabbagh, Andravizo \& Johnson, 2009; Libertus \& Needham, 
2014). Moreover, like adults, infants respond differently to upright faces compared to an inverted face (Cashon \& Holt, 2015). By around 7 months of age, infants recognize an individual face by the specific combination of eyes, nose, and mouth, but only if the face is upright (Cohen \& Cashon, 2001). However, younger infants show no difference in the way they process upright and inverted faces (Cashon \& Cohen, 2004). It is clear from this research that faces-specifically upright faces--are significant to infants and that an adult-like face expertise in processing has developed by 7 months. This bears the question of pareidolia. Is it possible that pareidolia occurs in infancy?

There are only two known studies that have tested this question (Otsuka, Hill, Kanazawa, Yamaguchi \& Spehar, 2012; Kobayashi, Otsuka, Nakato, Kanazawa, Yamaguchi, \& Kakigi, 2012). Both studies used a preferential looking paradigm to determine if infants perceived faces in the stimuli. In these studies, infants were shown upright and inverted versions of a face-like stimulus side by side on a monitor. It was reasoned by the researchers that because infants prefer looking at upright faces over inverted faces, infants would show a preference for the upright image if they perceived a face in the stimulus. In one study of 3-4 month olds, Otsuka et al. (2012) examined whether infants perceived a face in Mooney images. A Mooney image is a two toned ambiguous stimulus that can be perceived as a face or another image depending on how it is viewed. Otsuka et al. found that infants preferred the upright over inverted Mooney images, suggesting that they perceived the faces in those ambiguous stimuli. In a second study, Kobayashi et al. (2012) investigated 7-8 month-old infants' perception of Arcimboldo paintings. These paintings are images of fruits and vegetables arranged to resemble a face if viewed in one orientation and a bowl of fruits and vegetables if viewed in the other orientation. Kobayashi et al. found the infants showed a preference for the orientation that resembled a face. Together, these 
two studies suggest that infants may experience pareidolia. However, the stimuli used in these studies were designed to look like faces. One question that remains is would infants detect faces in stimuli that appear less face-like?

In the present study, we addressed this question by testing 7-9 month-old infants on their ability to see a face in stimuli that ranged from a realistic face image to more ambiguous, less face-like stimuli. Eight different stimuli were selected to be presented to participants (see Figure 1). The first stimulus was a realistic human face obtained from the NimStim database (Tottenham et al., 2009). We chose this stimulus as a control. At the age of 7 months infants should exhibit a preference for upright over inverted real human faces (for review, see Cashon \& Holt, 2015). If our results were to show that infants did not prefer the upright version of this face compared to the inverted one then we would know there was a flaw in our setup.

The second stimulus we selected was a Schematic face. This image resembles a paddle with the features of a smiling face on it (e.g. eyes, nose, and mouth). This stimulus has been used in a number of studies with infants to examine whether newborns and infants have a preference for face-like images (Goren, Sarty, \& Wu, 1975; Johnson, Dziurawiec, Ellis \& Morton, 1991). For example, in a classic study by Goren, Sarty and Wu (1975), the experimenters presented four different versions of the Schematic face to newborns: the original schematic, a moderately scrambled face, a scrambled face and a blank face. They measured how far the neonates would turn their heads and their eyes in order to follow the images as they moved. The researchers found infants followed the original Schematic face significantly farther than the other three stimuli. This and other similar findings have been taken as evidence that newborns have a preference for face-like images. 
Based on the previous research by Kobayashi et al. (2012) and Otsuka at al. (2012) we also included a Mooney face and an Arcimboldo painting. The Mooney face chosen is a twotoned image that, when viewed in the upright orientation, can be viewed as a female face or a profile of a man playing a saxophone. The Arcimboldo painting that was chosen was one of the stimuli used by Kobayashi et al. In one orientation, it looks like a bowl of vegetables; in the other, the collection of vegetable resembles a face.

The four remaining stimuli were chosen because they are less clearly face-like. The fifth stimulus is a grouping of trees that subtly outlines the features of a female face. The sixth is a photograph of a boulder with carvings that resemble the profile view of a man's face. The seventh is a photo of a cloud with shadows that could be perceived as two eyes and a mouth. The last stimulus is a Gaussian noise image that has slight shadows where eyes and a mouth would be in a face.

To estimate how adults viewed these images, 13 adults were shown photographs of the stimuli and asked to order them from "most like a face to least like a face." The mean rankings were calculated for each stimulus. The order of face-likeness based on the mean rankings was: Realistic Face $(M=1, S D=0)$, Schematic Face $(M=2.54, S D=1.03)$, Tree $(M=3.15, S D=$ $1.25)$, Mooney $(M=4.00, S D=0.77)$, Vegetable $(M=5.00, S D=1.43)$, Rock $(M=6.00, S D=$ 0.77), Cloud $(M=6.62, S D=0.83)$, Gaussian Noise $(M=7.69, S D=0.67)$.

There are several possible outcomes for this study. Based on previous research with infants, we predict that infants will show an upright preference for the realistic human face, schematic face, Mooney, and Arcimboldo. Additionally, because adults ranked the tree face stimulus high, we thought it was possible that infants may perceive a face in the tree image as 
well. However, if infants are like adults and also experience pareidolia, infants should show an upright stimulus preference for all eight stimuli.

\section{Methods}

\section{Participants}

The study was conducted with 47 full-term healthy infants aged 7 to 9 months $(M=$ 7.91, $S D=0.75 ; 18$ male, 29 female $)$. Participants included 17 7-month-olds $(M=7.10 ; \mathrm{SD}=$ 0.22; 6 male, 11 female), 17 8-month-olds $(M=8.02 ; \mathrm{SD}=0.30 ; 7$ Male, 10 Female), and 139 month-olds $(M=8.84 ; \mathrm{SD}=0.28 ; 5$ Male, 8 Female $)$. An additional four infants were tested but not included for the following reasons: the infant was premature $(n=1)$, coding was unreliable $(n=1)$, experimenter error $(n=2)$. Infants were selected through voluntary participation. A list of infants born in the local metro area was provided by the Kentucky Cabinet for Health and Family Services. Letters of invitation were sent to parents of infants in the desired age range on this list. Infants were also recruited via word of mouth on Facebook. Participation was completely voluntary. Upon completion of the experiment, participants were given a small gift (e.g., bib or t-shirt) for volunteering.

\section{Procedure}

When participants arrived at the lab, they were brought to a room to go over the procedure of the study and sign the consent form. Participants were then taken to the testing room. Infants were seated on their parents' lap approximately $120 \mathrm{~cm}$ away from the presentation monitor, a Panasonic 50" color plasma screen (45 X 26 inches with 1024 X 576 resolution).

Parents were instructed not to talk or point during the experiment. Parents were also asked to try to keep their infants centered on their laps so the babies would stay in view of the video camera hidden beneath the monitor. Lights in the room were dimmed before testing began. 
In a separate room, the experimenter observed the infant on a 15 " JVC closed-circuit color monitor connected to a Canon VC-C50i camera, hidden beneath the monitor in the testing room. The experimenter used keyboard presses on a Macintosh Power Mac G5 system running Habit X1.0 software (Cohen, Atkinson, \& Chaput, 2004) to present stimuli.

Each testing session consisted of 16 ten-second trials. On each trial, infants were shown an upright and inverted version of the same stimulus side by side on the monitor. Infants saw each of the eight pairs of stimuli twice, once with the upright version on the left and again with the upright image on the right. Stimulus order was randomized across the sixteen trials.

To begin each testing session and capture the infant's attention, the experimenter presented a video of a looming green circle on a black background accompanied by a rattle noise. When the experimenter saw that the infant was looking toward the monitor, the experimenter pressed the "enter" key to start the first trial and present the first pair of images. After 10 seconds had passed, the attention-getting video automatically played again on the monitor. Once again, when the infant was looking toward the monitor, the experimenter would press “enter" to begin the next trial. This process was repeated for a total of 16 trials.

\section{Coding}

Each testing session was recorded and saved to a DVD in order to later code infant looking time offline using Datavyu (2014) coding software. Direction of eye gaze was coded frame by frame for each trial by coders who were blind to what the infant was viewing. Upright preference scores were calculated for each trial by taking infants' looking time toward the upright stimulus and dividing that by the total time the infant looked at the upright and inverted stimuli combined (Upright preferance score $\left.=\frac{\text { upright looking time }}{\text { upright looking time+inverted looking time }}\right)$. Mean 
upright preference scores were calculated for each stimulus type by averaging each infant's scores across the two presentations of each stimulus.

\section{Results}

The first step in the analysis was to determine if there were any significant effects of age on upright preference scores. To test this question, a $3 \times 8($ Age $[7,8,9] \times$ Stimulus [realistic face, schematic face, Mooney, vegetable, tree, rock, cloud, Gaussian noise]) mixed model ANOVA, with stimulus as a repeated measures variable, was conducted. A statistically significant main effect of stimulus was found, $F(7,308)=2.92, p=.006$. Paired t-tests indicated that mean upright preference scores were significantly higher for realistic face than tree $(t(46)=$ $2.21, p=.032)$, rock $(t(46)=2.87, p=.006)$ and Gaussian noise $(t(46)=2.15, p=.037)$.

However, in terms of age, no statistically significant effects was found; neither the main effect for age nor the Age $\times$ Stimulus interaction was found to be significant, $F(2,44)=1.65, p=.203$ and $F(14,308)=0.79, p=.677$, respectively. Therefore, the data were collapsed across age in the subsequent analyses.

The second set of analyses directly tested the hypothesis that infants would show an upright preference for stimuli in which they perceived a face. Mean upright preference scores for all eight stimuli are presented in Figure 2. A one sample t-test was conducted for each stimulus to compare infants' mean upright preference score against chance (50\% of the total looking time). Infants showed a statistically significant upright preference for three of the eight stimuli, realistic face $\mathrm{t}(46)=2.90, \mathrm{p}=.006 ;$ schematic face, $\mathrm{t}(46)=3.94, \mathrm{p}<.001 ;$ and Mooney, $\mathrm{t}(46)=$ $2.24, \mathrm{p}=.030$.

\section{Discussion}


Based on past infant research (Cashon \& Holt, 2015; Goren, Sarty, \& Wu, 1975; Johnson, Dziurawiec, Ellis \& Morton, 1991; Kobayashi et al., 2012; Otsuka et al., 2012), our primary hypothesis was that infants 7-9 months would detect a face in the upright version of the realistic face, schematic face, Mooney, and Arcimboldo images. Our results partially supported these hypotheses. Infants showed an upright preference for the realistic face, schematic face, and Mooney, but not for the Arcimboldo image.

Finding that infants showed an upright preference for the realistic face and schematic face was the least surprising result. These stimuli have been tested extensively in the infant face perception literature (Cashon \& Holt, 2015) and were ranked highest by the adults surveyed. They were considered to be the most face-like and least ambiguous of the eight stimuli.

The results with the next set of images were somewhat unexpected. Along with the tree image, the Mooney and vegetable faces were among the next three "face-like" stimuli ranked by adults. These three stimuli could be classified as moderately face-like compared to the other stimuli. Again, based on previous research with infants we hypothesized that infants would perceive a face in the upright Mooney and vegetable stimuli (Kobayashi et al., 2012; Otsuka et al., 2012). However, infants in our study showed a significant preference only for the upright Mooney image.

The reason why infants in our study did not replicate those of Kobayashi et al. (2012) with the Arcimboldo vegetable image is unclear. Differences in procedures between our two studies may be the cause. Kobayashi et al. had infants looking at pairs of images for two 20 second trials. In our study, infants viewed the Arcimboldo images for two 10 second trials. In addition, infants in our study viewed a total of 16 stimulus pairs, whereas infants in Kobayashi et al. only viewed 3 pairs. It is not entirely clear why these procedural differences should lead to 
different findings. One possibility is the longer experimental session time in our study may have led to infant fatigue. However, it is not clear why fatigue would have affected the results for the Arcimboldo image more so than the realistic face, schematic face or Mooney faces. It is also quite possible that the null result was a spurious finding, and if this experiment were repeated, the results with the Arcimboldo image would replicate those of Kobayashi et al.

Infants also did not show a significant preference for the upright tree stimulus. There has not been any previous infant research using this image, but adults surveyed ranked the tree stimuli as third most face-like. Because of this, we thought it was possible that infants could show an upright preference for the tree face image as well. However, we found no evidence of this. It is possible that the facial features in the image are not as salient as the dark tree branches on the outer edges of the image. Because of this, the inverted version of the stimulus may have been as visually intriguing to infants as the upright version.

The last three stimuli based on adult rankings were rock, cloud and Gaussian noise. We did not expect that infants would detect a face in any of these stimuli based on the low rankings from adults and the lack of previous infant research using these stimuli. In fact, we did not find any clear evidence that infants at 7-9 months perceived faces in these stimuli. It is not clear from this study if failing to see faces in these stimuli is unique to infants. In our survey of adults we asked adults to rank the stimuli from most like a face to least like a face. However, we did not ask adults to tell us whether or not they saw a face in the stimuli. Since adults were not asked if they could see a face, we cannot conclude that they actually saw faces in all of the stimuli. Further research should be conducted to determine if adults reliably see faces in these highly ambiguous stimuli as well. 
The present study is one of the first, to our knowledge, to test infants' perception of faces in a wide range of face-like stimuli. Determining an appropriate method of study was a significant challenge. Infants cannot verbally communicate with us to tell us exactly what they are seeing in stimuli; therefore we must interpret their actions and make assumptions about what those actions mean. Our solution was to build on the evidence that infants prefer upright faces. We varied the face-like-ness in the stimuli and then interpreted upright preferences as evidence that infants saw faces in those stimuli. However, admittedly this measure is somewhat indirect and our assumption may be incorrect. We cannot be completely sure that infants' preferences were the result of perceiving faces. There may be other ways we could test infants' abilities to see faces in the future. One possible technique would be to use eye-tracking technology to view where an infant is looking. We could present infants with a realistic scene that would include a face-like object in it. Visual scanning behaviors (e.g., where the infant looked on the screen and for how long) would then be used to see to see if infants were capable of distinguishing the face in the scene. Past research by Riby and Hancock (2009) suggests this may be a reliable way to study face detection. In a study using eye tracking technology to measure visual attention in an image with an embedded picture of a face, Riby and Hancock (2009) found that children with autism took significantly longer to locate the face than did typically-developing children. Typically-developing children took an average of $1,553 \mathrm{~ms}$ to locate a face, signifying that the face stood out quickly. Measuring time to detect a face or face-like image in a scene may be a useful way to test the same question with a different method.

Future studies should test infants using other images as well. We only used one type of Mooney face and one Arcimboldo image. We do not know if the results found are specific to the Mooney and Arcimboldo images we included in this study or if they generalize to other similar 
images. In addition, some of the stimuli that we used contained very subtle representations of faces. It is possible that if some of those more ambiguous stimuli were made more face-like then infants might have been able to see the faces in them.

In summary, we conclude that infants around 7-9 months can perceive a face in an image if it closely resembles a face. The results suggest that infants at this age may experience some form of pareidolia but not to the same extent as adults do. Whether or not older infants can detect faces in more ambiguous images is unknown. Future studies should further investigate the development of pareidolia in infants and children. Given that faces are so special to infants and their facial perception is undergoing many changes in the first year of life, it is important to continue researching the development of face perception including the phenomenon of pareidolia, in infants. 


\section{References}

Cashon, C. H., \& Holt, N. A. (2015). Developmental origins of the face inversion effect. In J. B. Benson (Ed.), Advances in Child Development and Behavior: Vol. 48 (pp. 117-150).

Cashon, C. H., \& Cohen, L. B. (2004). Beyond U-shaped development in infants' processing of faces: an information-processing account. Journal of Cognition \& Development, 5, 59-80.

Cohen, L. B., \& Cashon, C. H. (2001). Do 7-month-old infants process independent features or facial configurations? Infant and Child Development, 10, 83-92. doi:10.1002/icd.250

Cohen, L. B., Atkinson, D. J., \& Chaput, H. H. (2004). HabitX: A new program for obtaining and organizing data in infant perception and cognition studies (Version 1.0). Austin: Texas.

Datavyu Team (2014). Datavyu: A Video Coding Tool. Databrary Project, New York University. URL http://datavyu.org.

DeNicola, C. A., Holt, N. A., Lambert, A. J., \& Cashon, C. H. (2013). Attention-orienting and attention-holding effects of faces on 4- to 8-month-old infants. International Journal of Behavioral Development, 37, 143-147. doi:10.1177/0165025412474751

Di Giorgio, E., Méary, D., Pascalis, O., \& Simion, F. (2013). The face perception system becomes species-specific at 3 months: An eye-tracking study. International Journal of Behavioral Development, 37, 95-99. doi:10.1177/0165025412465362

Gliga, T., Elsabbagh, M., Andravizou, A., \& Johnson, M. (2009). Faces attract infants' attention in complex displays. Infancy, 14, 550-562. doi:10.1080/15250000903144199

Goren, C. C., Sarty, M., \& Wu, P. Y. (1975). Visual following and pattern discrimination of face-like stimuli by newborn infants. Pediatrics, 56(4), 544. 
Guo, K., Meints, K., Hall, C., Hall, S., \& Mills, D. (2009). Left gaze bias in humans, rhesus monkeys and domestic dogs. Animal Cognition, 12, 409-418. doi:10.1007/s10071-008$0199-3$

Ichikawa, H., Kanazawa, S., \& Yamaguchi, M. K. (2011). Finding a face in a face-like object. Perception, 40, 500-502. doi: 10.1068/p6926

Jakobsen, K. V., Umstead, L., \& Simpson, E. A. (2016). Efficient human face detection in infancy. Developmental Psychobiology, 58, 129-136. doi:10.1002/dev.21338

Johnson, M. H., Dziurawiec, S., Ellis, H., \& Morton, J. (1991). Newborns' preferential tracking of face-like stimuli and its subsequent decline. Cognition, 40, 1-19.

Kanwisher, N. (2000). Domain specificity in face perception. Nature Neuroscience, 3, 759-763. doi:10.1038/77664

Kato, M. \& Mugitani, R. (2015) Pareidolia in Infants. PLoS ONE, 1O(2): e0118539. doi:10.1371/ journal.pone.0118539

Kobayashi, M., Otsuka, Y., Nakato, E., Kanazawa, S., Yamaguchi, M. K., \& Kakigi, R. (2012). Do infants recognize the Arcimboldo images as faces? Behavioral and near-infrared spectroscopic study. Journal of Experimental Child Psychology, 111, 22-36. doi:10.1016/j.jecp.2011.07.008

Libertus, K., \& Needham, A. (2014). Face preference in infancy and its relation to motor activity. International Journal of Behavioral Development, 38, 529-538. doi: $10.1177 / 0165025414535122$

Maurer, D., Le Grand, R., Mondloch, C. J. (2002). The many faces of configural processing. Trends in Cognitive Sciences, 6 (2002), pp. 255-260.

Otsuka, Y., Hill, H. C., Kanazawa, S., Yamaguchi, M. K., \& Spehar, B. (2012). Perception of 
Mooney faces by young infants: The role of local feature visibility, contrast polarity, and motion. Journal of Experimental Child Psychology, 111, 164-179.

doi:10.1016/j.jecp.2010.10.014

Rakover, S. S. (2013). Explaining the face-inversion effect: the face-scheme incompatibility (FSI) model. Psychonomic bulletin \& review, 20(4), 665-692.

Riby, D., \& Hancock, P. (2009). Do Faces Capture the Attention of Individuals with Williams Syndrome or Autism? Evidence from Tracking Eye Movements. Journal of Autism \& Developmental Disorders, 39, 421-431. doi:10.1007/s10803-008-0641-z

Tottenham, N., Tanaka, J., Leon, A. C., McCarry, T., Nurse, M., \& Hare, T. A., Nelson, C. (2009). The NimStim set of facial expressions: judgements from untrained research participants. Psychiatry Research, 168, 242-249. Doi: 10.1016/j.psychres.2008.05.006 

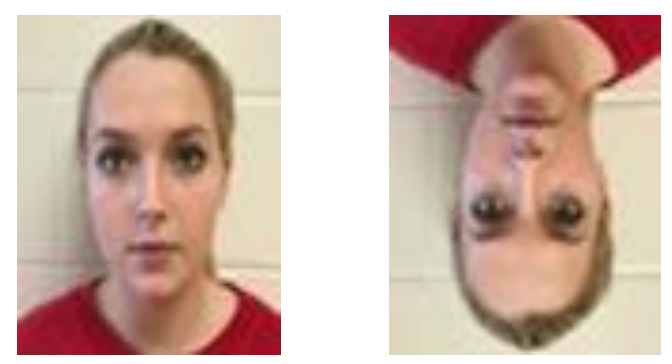

Realistic Face
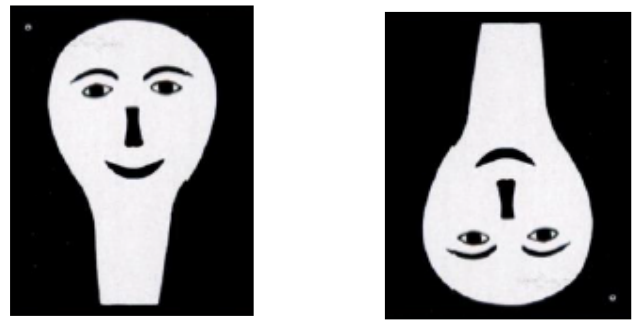

Schematic Face
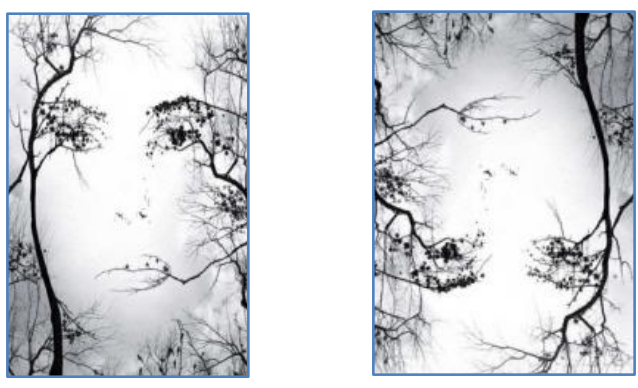

Tree

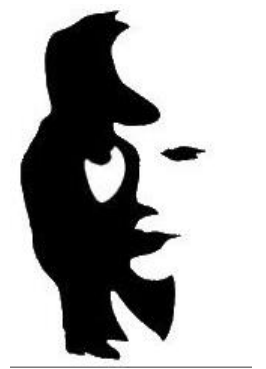

Mooney
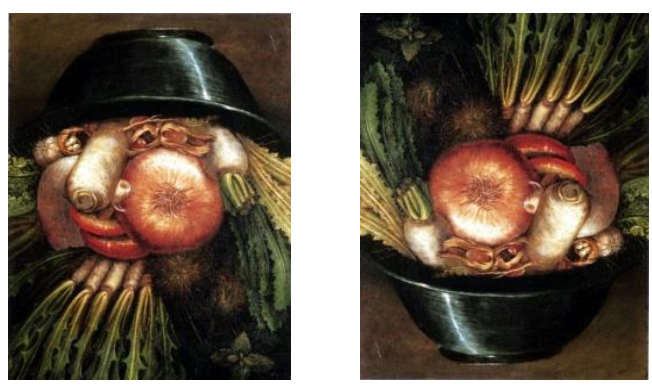

Vegetable
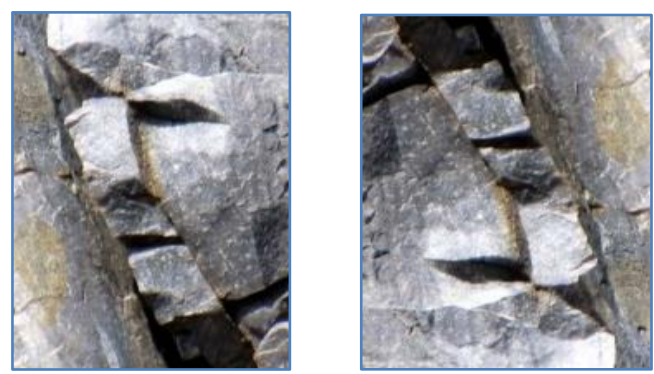

Rock
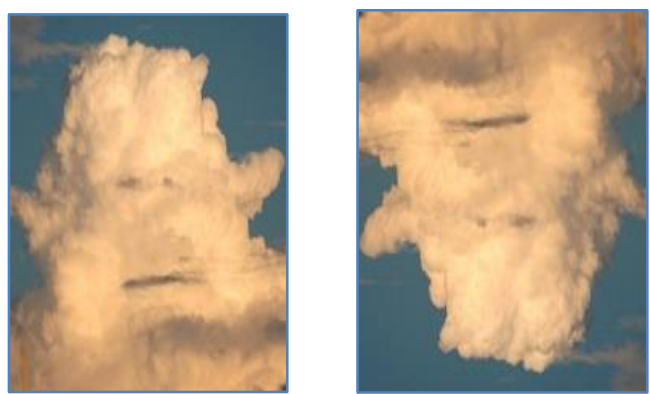

Cloud
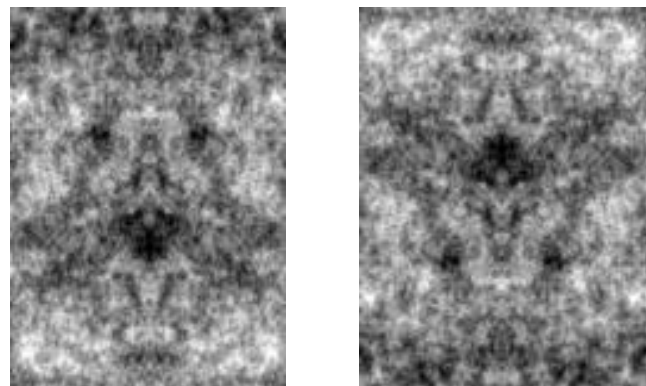

Gaussian Noise

Figure 1. Stimuli are shown here in the order adults ranked the upright stimuli as "most facelike" (top left) to "least face-like" (bottom right). The realistic face is not the actual stimulus used in the study. 


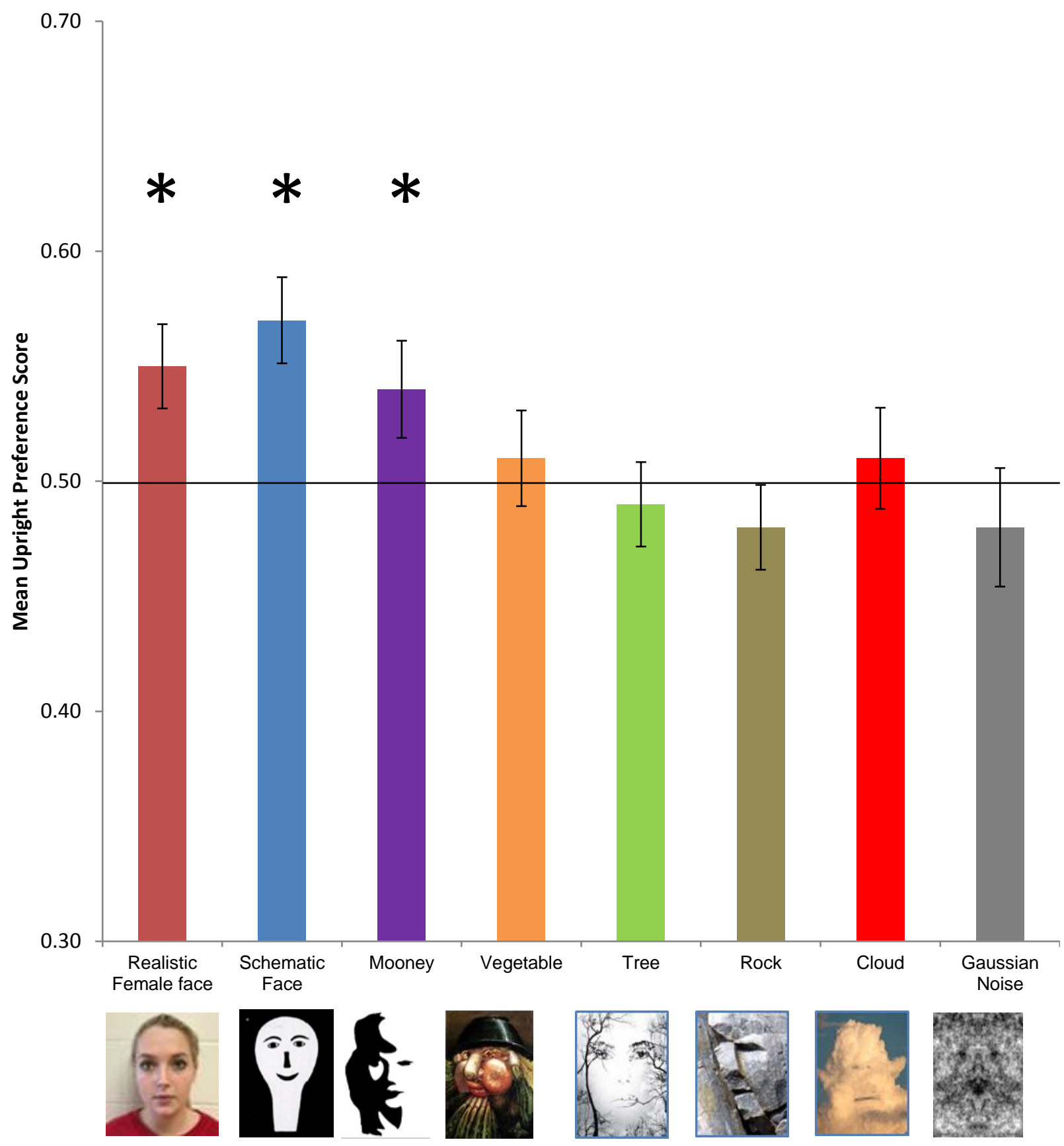

Stimuli

Figure 2. Infants' mean upright preference scores for each stimulus. Solid line depicting a preference score due to chance (.50). Error bars represent the standard error of the mean. 